\title{
Non-Cognitive Characteristics of Older Adults With Mild Cognitive Impairment And Errors On The Clock Drawing Test
}

Hiroyuki Umegaki ( $\square$ umegaki@med.nagoya-u.ac.jp )

Nagoya University Graduate School of Medicine

Yusuke Suzuki

Nagoya University Hospital

Hitoshi Komiya

Nagoya University Hospital

Kazuhisa Watanabe

Nagoya University Graduate School of Medicine

Masaki Nagae

Nagoya University Graduate School of Medicine

Yosuke Yamada

Nagoya University Graduate School of Medicine

Masafumi Kuzuya

Nagoya University Graduate School of Medicine

\section{Research Article}

Keywords: cognitive, CDT, qualitative, association,

Posted Date: November 16th, 2021

DOI: https://doi.org/10.21203/rs.3.rs-1038055/v1

License: (c) (i) This work is licensed under a Creative Commons Attribution 4.0 International License. Read Full License 


\section{Abstract}

Individuals with mild cognitive impairment $(\mathrm{MCl})$ often make qualitative errors on the Clock Drawing Test (CDT), and these errors are reported to be associated with lower scores on neuropsychological assessments. However, the association of CDT errors with non-cognitive characteristics has not been investigated in individuals with $\mathrm{MCl}$ to date. In this study, we explored this association in older adults with $\mathrm{MCl}$ who made qualitative errors on the CDT. Outpatients at a memory clinic with a Clinical Dementia Rating (CDR) of $0.5(n=193)$ were included. The CDT was evaluated using Cahn et al.'s method and noncognitive characteristics were compared between participants with and without CDT errors. The noncognitive characteristics compared included normal and maximum gait speeds, grip strength, muscle mass, and severity of white matter lesions.

Participants with the CDT error type of conceptual deficit (CD; $n=58)$ had significantly slower normal and maximum gait speeds, and multiple logistic regression analysis adjusted for age and sex revealed CD was significantly associated with the slowest tertile of maximum gait speed. No other non-cognitive characteristics were significantly associated with errors on the CDT.

\section{Introduction}

The Clock Drawing Test (CDT) has long been used as a screening test for dementia, and its validity has been confirmed. Several scoring systems for the CDT have been proposed (1). Quantitative scoring uses a numerical scale to assess the drawing, while qualitative scoring evaluates characteristic errors in drawing the clock. Several error types are identified in the qualitative scoring systems (1).

People with typical cognition also often make errors on the CDT. We previously reported that individuals with typical cognition and those with mild cognitive impairment ( $\mathrm{MCl}$ ) made CDT errors (3), evaluated using Cahn et al.'s method (2), and that these errors were associated with lower scores on neurocognitive assessments (3). We have also reported an association between the conceptual deficit (CD) error type on the CDT in non-demented older adults and subsequent progression to dementia (4).It is currently not known whether people with relatively preserved cognition, such as those with $\mathrm{MCl}$, who make CDT errors have common non-cognitive characteristics. This warrants investigation because we previously reported that physical function and cognitive function were closely associated, and declined in parallel, (5). Also, reduced gait speed is considered to be associated with cognitive impairment $(6 ; 7)$ and cognitive decline (8). Grip strength is also reported to be associated with cognition (9). Moreover, although the association between white matter lesions (WMLs) on magnetic resonance images (MRI) and cognitive decline is well established (10), and these lesions are reported to be associated with lower CDT scores (11), it is not known whether CDT errors are associated with WMLs.

In this study, we evaluated the non-cognitive characteristics of older adults with $\mathrm{MCl}$ who made errors on the CDT and examined the association of these characteristics and the involvement of WMLs with the type of CDT errors made. 


\section{Methods}

\section{Participants}

Among participants attending a memory clinic at a university hospital in Japan, those with a Clinical Dementia Rating (CDR) score of 0.5 (12) were included. A CDR score of 0.5 has been used as a criterion for diagnosing $\mathrm{MCl}$ (13). All participants were Japanese. The exclusion criteria were current serious medical or psychiatric disorder, history of symptomatic cerebrovascular disease, and gait disturbance or needing an assistive device to walk. The study protocol was approved by the Ethics Committee of the Graduate School of Medicine, Nagoya University (2015-04356977). Written informed consent was obtained from all participants. All methods were performed in accordance with the relevant guidelines and regulations.

\section{CDR}

The CDR is widely used to determine the clinical stage of cognitive impairment $(12 ; 13)$. Ratings are assigned based on information received from the subject and an accompanying informant on six subscales (memory, orientation, home and hobbies, judgement and problem solving, community affairs, and personal care). Each subscale is rated on a scale of $0-3(0=$ no impairment, $3=$ need for maximal assistance), with a global CDR range of $0-3(0=$ a normal healthy individual with no cognitive or functional deficits; $0.5=$ a normal healthy individual but with questionable cognitive and/or functional abilities indicating $\mathrm{MCl} ; 1$ = mild dementia; 2 = moderate dementia; and 3 = severe dementia). Participants in this study had global CDR scores of either 0 or 0.5 .

Neuropsychological assessments

Three experienced clinical neuropsychologists administered the Mini Mental State Examination (MMSE) (14) and the Alzheimer's Disease Assessment Scale (ADAS) for general cognitive function (15).

\section{Clock Drawing Test}

The neuropsychologists administered the CDT. Participants were given a blank piece of paper and instructed to draw a clock face measuring $10 \mathrm{~cm}$ in diameter, write all the numbers on it, and then draw hands indicating a time of 10 past 11 . The neuropsychologists then independently scored the drawn clocks using the method of Cahn et al. (2), whereby both quantitative and qualitative scores are assessed independently. Quantitative scores were determined for three components-integrity of the clock face (02 points), absence and sequencing of the numbers ( $0-4$ points), and presence and sequencing of the hands (0-4 points) -and qualitative scores were determined for the eight error types: 1 ) stimulus-bound response (SB); 2) conceptual deficit (CD), where the error reflects a loss or deficit in accessing knowledge about the attributes, features, and meaning of a clock; 3 ) perseveration (PR), where the activity continues or recurs without an appropriate stimulus; 4) neglect of left hemi-space (NL), where the clock's attributes are written only on the right half of the clock face; 5) planning deficit (PD), where there are gaps before 
the numbers $12,3,6$ or $9 ; 6$ ) nonspecific spatial error (NS), where there is no specific pattern in the disorganization of the spatial layout of numbers; 7) numbers written outside of the clock (OC), where the numbers are written either on or around the perimeter; and 8) numbers written counterclockwise (CC), where the numbers are arranged with 12 at the top and then continuing counterclockwise (2). Total CDT score was calculated by subtracting the sum of qualitative scores from the sum of quantitative scores, with a maximum score of 10 . The higher the score, the better the performance on the CDT. Cronbach' alpha for the three raters was 0.980 .

\section{Physical assessments}

Grip strength in the participant's dominant hand was measured using a portable grip strength dynamometer (GRIP-D; Takei, Niigata, Japan). Gait speed (m/s) was measured using a stopwatch. Participants were instructed to walk $5 \mathrm{~m}$ on a flat, straight path twice at their preferred speed and twice at maximum speed, with the average times calculated for each. Two markers were used to indicate the start and end of the walking path. Participants walked a 1-m section in advance of the start marker to ensure that they were walking at a comfortable pace by the time they reached it and the stopwatch was started.

Body composition analysis

The amounts of extracellular and intracellular water in the body were directly measured using electrical current generated at three frequencies $(5,50$, and $250 \mathrm{kHz})$ using a bioelectrical impedance data acquisition system (Inbody 430; Biospace Co., Seoul, South Korea). The system determines appendicular skeletal muscle mass from the segmental body composition and muscle mass. The skeletal muscle mass index (SMI) was calculated by dividing participants' muscle mass by their height in meters squared $\left(\mathrm{kg} / \mathrm{m}^{2}\right)$.

Brain MRI

T2-weighted and axial FLAIR sequence images were obtained for all participants by head MRI using a 1.5-T MR scanner (either a Vantage system [Toshiba Medical Systems Corporation, Otawara, Japan] or an Avanto system [Siemens, Munich, Germany]). The slice settings were as follows: 20 slices with 6.0$\mathrm{mm}$ thickness and an interslice gap of 7.2-7.5 mm obtained in parallel with the anterior commissureposterior commissure line, covering a maximum of 144-150 mm and ranging from the vertex to the lower end of the pons. Eight-channel head coils were used for MR signal acquisition and transmission. The parameters of the T2-weighted sequence were as follows: repetition time, 4000-4500 ms; echo time, 91 $105 \mathrm{~ms}$; echo train, 13; and number of excitations, 1. The parameters of the FLAIR sequence were as follows: repetition time, $8000 \mathrm{~ms}$; echo time, $100 \mathrm{~ms}$; and inversion time, $2500 \mathrm{~ms}$.

\section{Evaluation of WMLs}

In this study, the severity of cerebral WMLs was evaluated using the Fazekas scale, the most widely used method for visually assessing the severity of age-related WMLs. There are four stages of severity (grades 
0-3) (16). WMLs were divided into 2 groups: paraventricular hyperintensities (PVH) and deep white matter hyperintensities (DWMH).

Statistical analysis

Continuous variables were compared using Student's T test, and categorical variables were compared using the chi-square test, between groups with or without each CDT error type. Multiple logistic regression analysis adjusted for age and sex was performed to examine the association of CDT errors with the lowest tertiles of normal and maximum gait speeds.

\section{Results}

The CDT error types of SB, CD, and PD occurred most frequently $(24.5 \%, 29.6 \%$, and $30.1 \%$, respectively) among the error types (PR, 3.1\%; NS, 8.7\%; OC, 6.1\%; and CC, 1.5\%; Fig. 1). None of the participants made NL errors.

When the non-cognitive characteristics were compared between participants with and without each error type, participants who made SB errors were significantly older and had lower general cognition (determined by MMSE and ADAS-J cog) and slightly slower gait speeds (Table 1). The CD error type was associated with fewer years of education and lower general cognition, and participants with this type of error had significantly slower normal and maximum gait speeds, although the differences were relatively small (Table 2). Female participants tended to make PD errors (Table 3). None of the error types were associated with $\mathrm{PVH}$ or DWMH findings on MRI.

The results of a multiple logistic regression analysis adjusted for age and sex to investigate the association of errors with the lowest tertile of normal and maximum gait speeds are shown in Table 4 . The CD error type was significantly associated with the lowest tertile of maximum gait speed (odds ratio $=2.16695 \% \mathrm{Cl}, 1.093-4.291)$.

\section{Discussion}

In this study we found that participants with $\mathrm{MCl}$ (CDR 0.5) who made the CD type of error on the CDT had significantly slower gait speed than those participants who did not make the error. Grip and muscle mass were not associated with errors on the CDT. WMLs were not associated with CDT errors.

The CD error refers to loss of knowledge about the features and meaning of a clock. Impairment of semantic memory is considered to be associated with this type of error, and the impairment is thought to be caused by dysfunction of the lateral temporal lobe (17). A study using single-photon emission computed tomography suggested that CD may be associated with lower blood perfusion in certain brain regions, including the posterior entorhinal cortex, posterior cingulate cortex, and parahippocampal cortex (18). Our previous study suggested that making the CD error was associated with the risk of developing dementia (4). In that study, CD was associated with impairment in several cognitive domain in both non- 
demented older adults and those with Alzheimer's Disease. In a review paper, Amodeo et al. concluded that among the eight CDT error types, CD errors reflect cognitive decline over time most effectively (19). Slow gait is an established risk factor for dementia (20), and the present finding that participants who made $C D$ errors had slower gait speed may be one of underlying mechanisms that $C D$ predicted cognitive decline in our previous study. Our previous study found that making CD errors was associated with lower executive function scores in individuals with $\mathrm{MCl}$ (3). Executive dysfunction is also reported to be associated with slow gait speed (21). Slow gait and executive dysfunction are also associated with falls (22), and CD errors on the CDT were found to be associated with falls in older adults (23).

Multiple logistic regression analysis revealed that only CD was significantly associated with the slowest tertile of maximum gait speed in this study. This is in agreement with a previous study where maximum gait speed showed better sensitivity than normal gait speed to discriminate lower cognitive status in community-dwelling older adults (24) and another study where maximum gait speed, but not normal gait speed, predicted cognitive decline (25). We also previously reported that maximum gait speed was associated with wider range of neuropsychological assessments than normal gait speed was (6).

In this study we used CDR 0.5 to define MCl, however, previously we used Petersen's criteria (26) to define it (4). Both sets of criteria have been widely used for the diagnosis of $\mathrm{MCl}$, and the population defined by these sets of criteria is reported to overlap but is not exactly the same $(27 ; 28)$. Therefore, the association between slower gait speed and $\mathrm{MCl}$ seen in our participants with $\mathrm{CD}$ error needs to be interpreted with caution.

In a systematic review, Supasitthumrong et al. concluded that the severity of WMLs was associated with lower CDT scores (29). However, to our knowledge, the present study is the first to have investigated this association and we found no association between severity of WMLs and the most frequent CDT errors in this study. We did not quantitatively measure WMLs in this study but used a semi-quantitative method. More objective quantification to estimate the severity of WMLs may alter the findings and should be investigated in future studies. Other limitations of our study are that we confined our evaluation to older adults with $\mathrm{MCl}$ (CDR 0.5). To clarify changes in CDT performance over time, older adults with dementia as well as those with typical cognition need to be included in further studies.

In conclusion, we found that older adults with $\mathrm{MCl}$ who made $\mathrm{CD}$ errors on the CDT had significantly slower gait speed, especially slower maximum gait speed.

\section{Declarations}

Author Contributions: "Conceptualization, HU.; methodology, HU; validation, YS, HK, KW.; formal analysis, $\mathrm{HU}$;; investigation, $\mathrm{HU}$; writing-original draft preparation, HU; writing-review and editing, YS.; supervision, MK.; All authors have read and agreed to the published version of the manuscript." 
Funding: This research was funded by the Grant Program of Innovation for Future Society of Nagoya University.

Acknowledgments: The authors thank Ms Kana Ogawa, Nozomi Ando, Rika Yamamoto for their technical support.

Conflicts of Interest: The authors declare no conflict of interest.

\section{References}

1. Spenciere, B. Alves, H. \&Charchat-Fichman, H. Scoring systems for the Clock Drawing Test: A historical review. Dement Neuropsychol. 11,6-14. https://doi.org/10.1590/1980-57642016dn11010003(2017).

2. Cahn, D.A. et al. Screening for dementia of the Alzheimer type in the community: The utility of the Clock Drawing Test. Arch Clin Neuropsychol. 11, 529-539(1996).

3. Umegaki, H. et al. Frequencies and Neuropsychological Characteristics of Errors in the Clock Drawing Test. J Alzheimers Dis. 82,1291-1300. https://doi.org/10.3233/JAD-210456(2021).

4. Umegaki, H. et al. Association of the Qualitative Clock Drawing Test with Progression to Dementia in Non-Demented Older Adults. J Clin Med 9,2850. https://doi.org/ 10.3390/jcm9092850(2020).

5. Fujisawa, C. et al. Physical Function Differences Between the Stages From Normal Cognition to Moderate Alzheimer Disease. J Am Med Dir Assoc. 18, 368.e9-368.e15. https://doi.org/ 10.1016/j.jamda.2016.12.079. (2017).

6. Umegaki, $\mathrm{H}$. et al. Maximum gait speed is associated with a wide range of cognitive functions in Japanese older adults with a Clinical Dementia Rating of 0.5. Geriatr Gerontol Int. 18,13231329. https://doi.org/ 10.1111/ggi.13464. (2018).

7. Peel, N.M., Alapatt, L.J., Jones, L.V., \& Hubbard, R.E. The Association Between Gait Speed and Cognitive Status in Community-Dwelling Older People: A Systematic Review and Meta-analysis. J Gerontol A Biol Sci Med Sci. 74,943-948. https://doi.org/10.1093/gerona/gly140(2019).

8. Grande, G. et al. Measuring gait speed to better identify prodromal dementia. Exp Gerontol.124,110625. https://doi.org/10.1016/j.exger.2019.05.014(2019).

9. Shaughnessy, K.A. et al. A Narrative Review of Handgrip Strength and Cognitive Functioning: Bringing a New Characteristic to Muscle Memory. J Alzheimers Dis. 73,1265-1278. https://doi.org/10.3233/JAD-190856(2020).

10. Cannistraro, R.J. et al. CNS small vessel disease: A clinical review. Neurology. 92,1146-1156. https://doi.org/10.1212/WNL.0000000000007654(2019).

11. Supasitthumrong, T., Herrmann, N., Tunvirachaisakul, C., \&Shulman, K. Clock drawing and neuroanatomical correlates: A systematic review. Int J Geriatr Psychiatry. 34,223-232. https://doi.org/10.1002/gps.5013(2019). 
12. Morris, J.C. The Clinical Dementia Rating (CDR): current version and scoring rules. Neurology.43,2412-4. https://doi.org/10.1212/wnl.43.11.2412-a(1993).

13. Morris, J.C. et al. Mild cognitive impairment represents early-stage Alzheimer disease. Arch Neurol. 58,397-405. https://doi.org/10.1001/archneur.58.3.397(2001).

14. Folstein, M.F., Folstein, S.E., \&McHugh, P.R. "Mini-mental state". A practical method for grading the cognitive state of patients for the clinician. J Psychiatr Res. 12,189-198.

https://doi.org/10.1016/0022-3956(75)90026-6(1975).

15. Mohs, R.C. \& Cohen, L. Alzheimer's Disease Assessment Scale (ADAS). Psychopharmacol Bull. 24,627-628(1988).

16. Schmidt, R. et al. Magnetic resonance imaging white matter lesions and cognitive impairment in hypertensive individuals. Arch Neurol.48,417-20. https://doi.org/10.1001/archneur.1991.00530160087019(1991).

17. Budson, A.E. Understanding memory dysfunction. Neurologist. 15,71-79. https://doi.org/10.1097/NRL.0b013e318188040d(2009).

18. Duro, D. et al. Clock drawing test in mild cognitive impairment: Correlation with cerebral perfusion in single-photon emission computed tomography. Neuropsychology. 33,617-632. https://doi.org/10.1037/neu0000549(2019).

19. Amodeo, S., Mainland, B.J., Herrmann, N., \& Shulman, K.I. The Times They Are a-Changin': Clock Drawing and Prediction of Dementia. J Geriatr Psychiatry Neurol. 28,145-155. https://doi.org/10.1177/0891988714554709(2015).

20. Grande,G. et al. Measuring gait speed to better identify prodromal dementia. Exp Gerontol. 124,110625. https://doi.org/10.1016/j.exger.2019.05.014(2019).

21. Kearney, F.C., Harwood, R.H., Gladman, J.R., Lincoln, N., \& Masud, T. The relationship between executive function and falls and gait abnormalities in older adults: a systematic review. Dement Geriatr Cogn Disord. 36,20-35. https://doi.org/10.1159/000350031(2013).

22. Zhang, W. et al. Review of Gait, Cognition, and Fall Risks with Implications for Fall Prevention in Older Adults with Dementia. Dement Geriatr Cogn Disord. 48,17-29. https://doi.org/10.1159/000504340(2019).

23. Suzuki, Y. et al. Quantitative and Qualitative Analyses of the Clock Drawing Test in Fall and Non-Fall Patients with Alzheimer's Disease. Dement Geriatr Cogn Dis Extra. 9,381-388. https://doi.org/10.1159/000502089(2019).

24. Fitzpatrick, A.L. et al. Associations of gait speed and other measures of physical function with cognition in a healthy cohort of elderly persons. J Gerontol A Biol Sci Med Sci.62,1244-51. https://doi.org/10.1093/gerona/62.11.1244(2007).

25. Suzuki, H. et al. One-Year Change in the Japanese Version of the Montreal Cognitive Assessment Performance and Related Predictors in Community-Dwelling Older Adults. J Am Geriatr Soc. 63,18749. https://doi.org/10.1111/jgs.13595(2015). 
26. Petersen, R.C. et al. Current concepts in mild cognitive impairment. Arch. Neurol. 58, 1985-1992. https://doi.org/ 10.1001/archneur.58.12.1985(2001).

27. Saxton, J.et al. Functional and cognitive criteria produce different rates of mild cognitive impairment and conversion to dementia. J Neurol Neurosurg Psychiatry. 80,737-43. https://doi.org/10.1136/jnnp.2008.160705(2009).

28. Ganguli, M. et al. Outcomes of mild cognitive impairment by definition: a population study. Arch Neurol. 68,761-7. https://doi.org/10.1001/archneurol.2011.101(2011).

29. Supasitthumrong, T., Herrmann, N., Tunvirachaisakul, C., \& Shulman, K. Clock drawing and neuroanatomical correlates: A systematic review. Int J Geriatr Psychiatry. 34,223-232. https://doi.org/10.1002/gps.5013(2019).

\section{Tables}

Table 1 Comparison of participant characteristics with or without SB error on the Clock Drawing Test

\begin{tabular}{|c|c|c|c|}
\hline & SB (+) & SB (-) & $\mathrm{p}$ value \\
\hline number & 48 & 148 & \\
\hline age (yeas) & $80.0 \pm 5.1$ & $77.9 \pm 7.3$ & 0.03 \\
\hline male (\%) & $50.00 \%$ & $42.60 \%$ & 0.23 \\
\hline educational years & $12.3 \pm 2.9$ & $12.6 \pm 2.8$ & 0.28 \\
\hline ADAS-J cog & $12.4 \pm 4.1$ & $9.4 \pm 4.4$ & $<0.01$ \\
\hline MMSE & $24.0 \pm 2.6$ & $26.0 \pm 2.6$ & $<0.01$ \\
\hline GDS-15 & $4.2 \pm 3.0$ & $4.0 \pm 3.0$ & 0.36 \\
\hline $\operatorname{BMI}\left(\mathrm{Kg} / \mathrm{m}^{2}\right)$ & $22.2 \pm 3.0$ & $22.6 \pm 3.4$ & 0.24 \\
\hline $\mathrm{SMI}\left(\mathrm{Kg} / \mathrm{m}^{2}\right) \quad$ male & $7.4 \pm 0.9$ & $7.3 \pm 0.7$ & 0.65 \\
\hline female & $5.6 \pm 0.6$ & $6.4 \pm 1.2$ & 0.31 \\
\hline Maximum gait speed $(\mathrm{m} / \mathrm{s})$ & $1.40 \pm 0.35$ & $1.46 \pm 0.35$ & 0.16 \\
\hline Normal gait speed (m/s) & $0.97 \pm 0.23$ & $1.01 \pm 0.23$ & 0.18 \\
\hline male & $27.3 \pm 6.9$ & $28.9 \pm 6.6$ & 0.17 \\
\hline female & $16.4 \pm 4.8$ & $17.3 \pm 4.7$ & 0.23 \\
\hline PVH (\%) $(\mathrm{N}=146)(0 / 1 / 2 / 3)$ & $70.0 \%(12 / 27 / 1 / 0)$ & $67.0 \%(35 / 63 / 7 / 1)$ & 0.65 \\
\hline DWMH $(\%)(N=146)(0 / 1 / 2 / 3)$ & $55.5 \%(18 / 20 / 2 / 0)$ & $49.1 \%(54 / 46 / 6 / 0)$ & 0.77 \\
\hline
\end{tabular}


ADAS: Alzheimer's Disease Assessment Scale; DWMH: deep white matter hyperintensities; MMSE: Mini Mental State Examination; PVH: paraventricular hyperintensities; SB: stimulus-bound response

Table 2 Comparison of participant characteristics with or without CD error on the Clock Drawing Test

\begin{tabular}{|c|c|c|c|}
\hline & $\mathrm{CD}(+)$ & $\mathrm{CD}(-)$ & $\mathrm{p}$ value \\
\hline number & 58 & 138 & \\
\hline age (yeas) & $79.1 \pm 6.8$ & $78.1 \pm 6.9$ & 0.17 \\
\hline male $(\%)$ & $43.10 \%$ & $44.90 \%$ & 0.47 \\
\hline educational years & $11.8 \pm 2.7$ & $12.8 \pm 2.8$ & 0.01 \\
\hline ADAS-J cog & $11.5 \pm 5.1$ & $9.5 \pm 4.2$ & 0.01 \\
\hline MMSE & $24.5 \pm 3.0$ & $25.9 \pm 2.5$ & $\nabla 0.01$ \\
\hline GDS-15 & $4.6 \pm 3.3$ & $3.8 \pm 2.8$ & 0.06 \\
\hline BMI $\left(\mathrm{Kg} / \mathrm{m}^{2}\right)$ & $22.8 \pm 4.0$ & $22.4 \pm 3.0$ & 0.21 \\
\hline $\mathrm{SMI}\left(\mathrm{Kg} / \mathrm{m}^{2}\right) \quad$ male & $7.2 \pm 1.0$ & $7.3 \pm 0.7$ & 0.25 \\
\hline female & $5.7 \pm 0.8$ & $5.7 \pm 0.7$ & 0.44 \\
\hline Maximum gait speed (m/s) & $1.35 \pm 0.33$ & $1.49 \pm 0.34$ & 0.01 \\
\hline Normal gait speed (m/s) & $0.96 \pm 0.22$ & $1.02 \pm 0.23$ & 0.04 \\
\hline male & $27.7 \pm 7.1$ & $28.7 \pm 6.5$ & 0.26 \\
\hline female & $17.0 \pm 4.5$ & $17.1 \pm 4.9$ & 0.45 \\
\hline $\mathrm{PVH}(\%)(\mathrm{N}=146)(0 / 1 / 2 / 3)$ & $76.7 \%(10 / 28 / 4 / 1)$ & $67.0 \%(35 / 63 / 7 / 1)$ & 0.65 \\
\hline DWMH $(\%)(N=146)(0 / 1 / 2 / 3)$ & $46.5 \%(23 / 18 / 2 / 0)$ & $52.4 \%(49 / 48 / 6 / 0)$ & 0.80 \\
\hline
\end{tabular}

ADAS: Alzheimer's Disease Assessment Scale; CD: conceptual deficit; DWMH: deep white matter hyperintensities; MMSE: Mini Mental State Examination; PVH: paraventricular hyperintensities

Table 3 Comparison of participant characteristics with or without PD error on the Clock Drawing Test 


\begin{tabular}{|llll|}
\hline & $\mathrm{PD}(+)$ & $\mathrm{PD}(-)$ & p value \\
\hline number & 59 & 137 & \\
\hline age (yeas) & $77.4 \pm 6.6$ & $78.8 \pm 7.0$ & 0.10 \\
\hline male (\%) & $27.10 \%$ & $51.80 \%$ & $\varangle 0.01$ \\
\hline educational years & $11.9 \pm 2.5$ & $12.8 \pm 2.9$ & 0.03 \\
\hline ADAS-J cog & $9.8 \pm 4.6$ & $10.2 \pm 4.5$ & 0.29 \\
\hline MMSE & $25.4 \pm 2.9$ & $25.6 \pm 2.7$ & 0.37 \\
\hline GDS-15 & $4.1 \pm 2.9$ & $4.0 \pm 3.0$ & 0.46 \\
\hline BMI (Kg/m ${ }^{2}$ male & $21.9 \pm 3.5$ & $22.8 \pm 3.2$ & 0.04 \\
\hline SMI(Kg/m $\left.{ }^{2}\right)$ & $7.4 \pm 0.9$ & $7.3 \pm 0.8$ & 0.28 \\
\hline \multicolumn{1}{c}{ female } & $5.6 \pm 0.7$ & $5.8 \pm 0.7$ & 0.08 \\
\hline Maximum gait speed (m/s) & $1.43 \pm 0.28$ & $1.46 \pm 0.37$ & 0.31 \\
\hline Normal gait speed (m/s) & $1.03 \pm 0.21$ & $0.99 \pm 0.24$ & 0.10 \\
\hline Grip male & $29.9 \pm 7.2$ & $28.1 \pm 6.5$ & 0.17 \\
\hline female & $16.3 \pm 4.8$ & $17.6 \pm 4.7$ & 0.08 \\
\hline PVH (\%) (N=146) (0/1/2/3) & $72.3 \%(13 / 30 / 3 / 1)$ & $65.7 \%(34 / 60 / 5 / 0)$ & 0.44 \\
\hline DWMH (\%) (N=146) (0/1/2/3) & $51.1 \%(23 / 21 / 3 / 0)$ & $50.5 \%(49 / 45 / 5 / 0)$ & 0.95 \\
\hline
\end{tabular}

ADAS: Alzheimer's Disease Assessment Scale; DWMH: deep white matter hyperintensities; MMSE: Mini Mental State Examination; PD: planning deficit; PVH: paraventricular hyperintensities

Table 4 Association of errors with the slowest tertiles of normal and maximum gait speeds determined by multiple logistic regression analysis adjusted for age and sex

\begin{tabular}{llllllll} 
& \multicolumn{3}{c}{ normal gait speed } & & maximum gait speed & \\
\hline & Odds ratio & $95 \% \mathrm{Cl}$ & $\mathrm{p}$ value & Odds ratio & $95 \% \mathrm{Cl}$ & $\mathrm{p}$ value \\
\hline SB & 1.568 & $0.768-3.202$ & 0.217 & 0.987 & $0.471-2.068$ & 0.973 \\
\hline CD & 1.323 & $0.667-2.627$ & 0.423 & 2.166 & $1.093-4.291$ & 0.027 \\
\hline PD & 0.901 & $0.427-1.897$ & 0.783 & 0.691 & $0.323-1.479$ & 0.342
\end{tabular}

adjusted with age and sex

SB: Stimulus-bound response; CD: Conceptual deficit; PD: Planning deficit; Cl: confidential interval 
Figures

\section{Frequencies of errors}

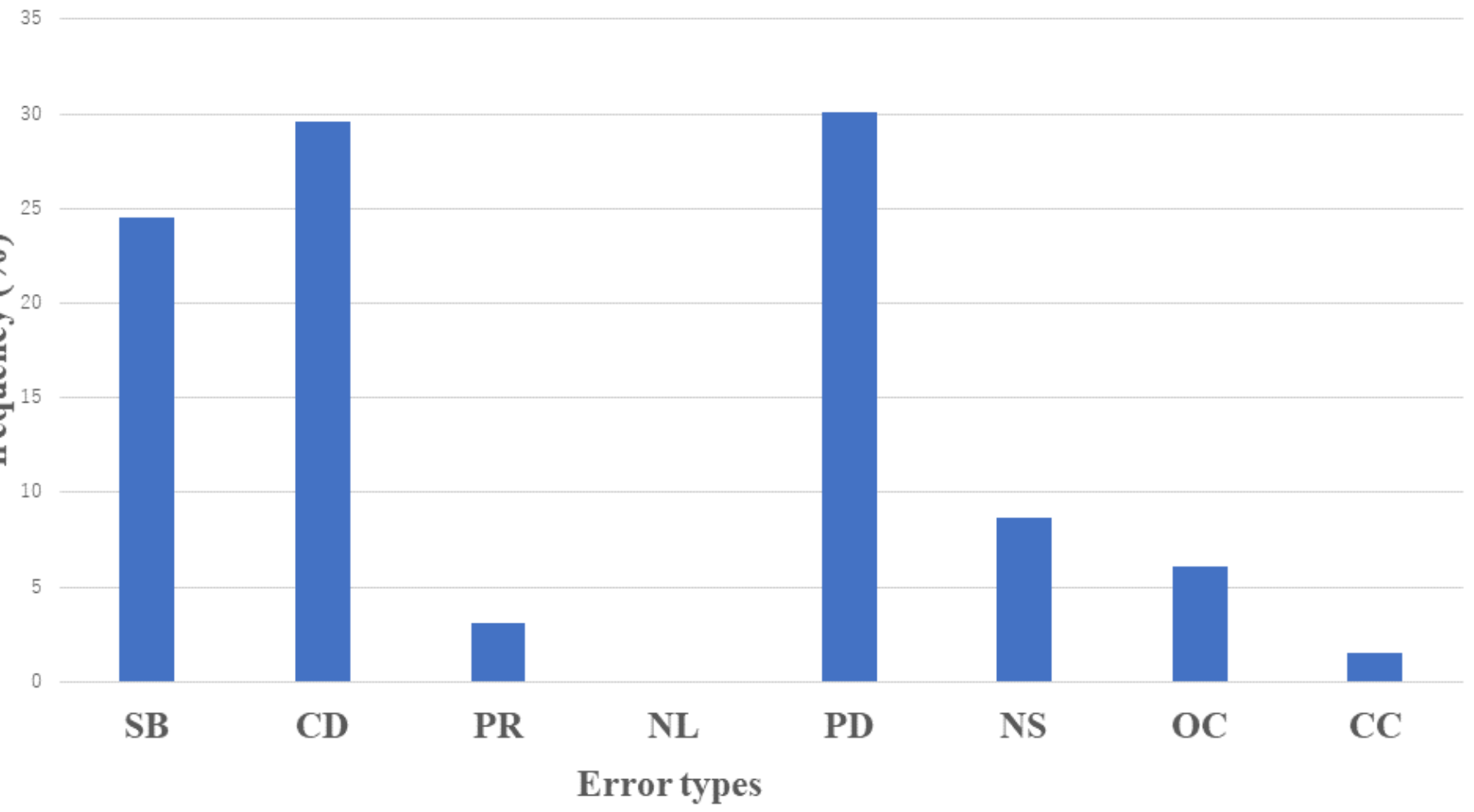

\section{Figure 1}

Frequencies of type of errors on the Clock Drawing Test SB: Stimulus-bound response; CD: conceptual deficit; PR: perseveration; NL: neglect of left hemi-space; PD: planning deficit; NS: nonspecific spatial error; OC: numbers written on outside of the clock; CC: numbers written counterclockwise 